\title{
A new method for SAR image coregistration from interferometric coherence modeling
}

\author{
Haiqin Cheng ${ }^{1,2}$, Qiang Chen ${ }^{1}$, Guoxiang Liu ${ }^{1}$, Yinghui Yang ${ }^{1}$ \\ 1. Department of Remote Sensing and Geoinformation Engineering, Southwest Jiaotong University, Chengdu 610031, China \\ 2. School of Civil Engineering and Architecture, East China Jiaotong University, Nanchang 330013, China
}

\begin{abstract}
A new method on single-looking complex SAR images coregistration from interferometric coherence modeling is proposed to accurately seek homologous points. The set of Doppler, slant-range and ellipsoid equations are employed to compute the coarse matching position. The mathematical model of spatial coherence surface can be established using discrete correlation values of central pixel and its adjacent positions. The accurate matching pixel of homologous point can be derived through the maximization operation of modeled mathematical coherence surface. The proposed interferometric coherence modeling method can greatly mitigate the coherence loss due to discrete searching windows in traditional SAR image coregistration algorithms. The ALOS/PALSAR acquisitions over the Longmenshan mountainous area in southwestern China are employed in the study. The testing results show that the coregistration accuracy of SAR images can reach $(0.1 \sim 0.01)$ pixels. The statistical interferometric coherence is improved with almost $5 \%$. The quality improvement of derived interferogram validates the reliability of the proposed SAR image coregistration method.
\end{abstract}

Index Terms-Interferometric coherence modeling, SAR image coregistration, InSAR, homologous pixel

\section{I . INTRODUCTION}

Interferometric Synthetic Aperture Radar (InSAR) has proven to be the efficient tool for generating large-area DEM and mapping ground deformation from two SAR complex images $[1,2]$. The SAR images are taken from slightly different orbital positions. One is the master image and the other as the slave image [3]. The co-registration between the master and slave images with the accuracy of sub-pixel is the important process for obtaining high quality fringes and accurate interferometric results $[4,5]$.

The existing methods for SAR image co-registration focus on the seeking of the pixel position with the highest coherence from searching area of slave image. Since the process is on the basis of discrete coherence windows search, there may be excessive or deficient movement that can lead to the matching error of homologous points.

In order to mitigate the coherence loss induced by the discrete window search in SAR image co-registration, an improved approach is proposed based on moving coherence surface fitting to detect accurate positions of homologous points. The slang-range and Doppler equations with satellite orbital state vector are first used to seek the coarse matched pixel $[6,7]$. The mathematical surface model is fitted based on the coherence values of central pixel and its adjacent pixels. The accurate positions of homologous points are obtained through the maximization of fitted coherence surface function.

\section{COARSE CO-REGISTRATION}

The coarse co-registration between master and slave images can be first carried out from the geometric relationship of satellite orbital positions, image point and ground point. The objective of coarse co-registration is to obtain the approximate offset vectors for the center pixel in master image. The slant-range, Doppler and ellipsoid equations are used to establish the spatial geometric model, expressed as the following $[1,7]$.

$$
\left\{\begin{array}{c}
\vec{R}=\vec{S}-\vec{P} \\
\vec{V} \cdot(\vec{S}-\vec{P})=0 \\
\frac{X^{2}}{a^{2}}+\frac{Y^{2}}{a^{2}}+\frac{Z^{2}}{b^{2}}=1
\end{array}\right.
$$

where $\vec{R}$ is the slant range vector, $\vec{S}$ and $\vec{V}$ are respectively the satellite orbital position and velocity vectors, $\vec{P}$ is the vector for the point $\mathrm{P}(X, Y, Z)$ on the ellipsoid, $a$ and $b$ are respectively the length of semimajor and semiminor axis of reference ellipsoid.

For the central pixel $\left(l_{m}, p_{m}\right)$ in master image, the set of equations is used to compute the coordinates of point $\mathrm{P}(X, Y, Z)$. According to the Doppler equation, the acquisition time $t$ of the matched point in the slave image can be figured out. The azimuth coordinate $l_{s}$ for the matched 
point is related to $t$ as follows $[1,2]$.

$$
l_{s}=\left(t-t_{0}\right) \cdot P R F+1
$$

where $t_{0}$ is the acquisition time of the first azimuth line of slave image, $P R F$ is the pulse repetition frequency in $\mathrm{Hz}$.

The slant range $R$ between the satellite orbital position and the point $\mathrm{P}(X, Y, Z)$ can be obtained. Thus the range number $p_{s}$ for the matched pixel in slave image can be written as the following $[1,2]$.

$$
p_{s}=1+2 \cdot R S R \cdot\left(R-R_{0}\right) / c
$$

where $R S R$ is the range sampling rate in $\mathrm{Hz}, R_{0}$ is the near slant range, $c$ is the light speed.

The approximate offset vectors in azimuth and range direction between the master and slave image can be figured out from the above method. It provides the initial offset value for the following fine co-registration. The accuracy of the coarse co-registration is about several to tens of pixels [1].

\section{COHERENCE SURFACE FITTING}

\subsection{Discrete coherence estimation}

A number of pixel locations (e.g. 200) are evenly selected in the master image, and the approximate matched points in the slave image could be obtained according to the above coarse offset. The cross-correlation of the selected pixel is further performed from the search window area of slave image. The coherence value of central pixel in the calculating window can be estimated as the following.

$$
\rho=\frac{\sum_{j=1}^{n} \sum_{i=1}^{m}\left(\left|s l c_{M}(i, j)\right| \cdot\left|s l c_{S}(i, j)\right|\right)}{\sqrt{\sum_{j=1}^{m} \sum_{i=1}^{n}\left|s l c_{M}\right|^{2} \cdot \sum_{j=1}^{m} \sum_{i=1}^{n}\left|s l c_{S}\right|^{2}}}
$$

where $s l c_{M}$ and $s l c_{S}$ are the master and slave complex image respectively, $m$ and $n$ are the size of the calculating window.

The discrete coherence field in the searching area is formed. The matched points with the estimated correlation peak can be found from the discrete coherence field. The co-registration accuracy of the discrete cross-correlation method is about one pixel.

\subsection{Continuous coherence surface}

Instead of using coherence interpolation, a new method based on moving coherence surface fitting is proposed. The fitted continuous surface model can mitigate the coherence loss due to discrete windows search in detecting homologous points. On the basis of the coherence values of central pixel and its adjacent pixels, the mathematical model of the moving coherence surface is given as the following $2 \mathrm{~d}$ polynomial.

$$
\rho(l, p)=c_{0}+c_{1} l+c_{2} p+c_{3} l^{2}+c_{4} l p+c_{5} p^{2}
$$

where $\rho$ is the coherence value, $c_{0}, \cdots, c_{5}$ are the coefficients of the fitted coherence function, $(l, p)$ are the coordinates in the azimuth and range directions.

From the coherence values at the central and its adjacent pixels in calculating windows, the coefficients for the fitted function can be estimated with the least squares method. So the fitting coherence surface model can be established. It should be noted that the image coordinates in the azimuth and range directions must be first normalized to avoid numerical instabilities.

In the fitted continuous coherence field, the maximum coherence value indicates the homologous point with high reliability. The maximum value of the $2 \mathrm{~d}$ coherence function could be calculated by taking the derivative of the variables $l$ and $p$ respectively as

$$
\left\{\begin{array}{l}
\frac{\partial \rho}{\partial l}=c_{1}+2 c_{3} l_{\max \rho}+c_{4} p_{\max \rho}=0 \\
\frac{\partial \rho}{\partial p}=c_{2}+2 c_{5} p_{\max \rho}+c_{4} l_{\max \rho}=0
\end{array}\right.
$$

Solving the above equations, the accurate matched positions $\left(l_{\max \rho}, p_{\max \rho}\right)$ at the peak of fitted coherence model is obtained with the co-registration accuracy of sub-pixel scale. Since the process is performed through the fast solution of differential equations, the calculation efficiency is also improved significantly.

The matched homologous points are employed to establish the coordinate transformation relationship between master and slave images with a two-dimensional polynomial as

$$
\left\{\begin{array}{l}
\Delta l=a_{1}+a_{2} l+a_{3} p+a_{4} l p+a_{5} l^{2}+a_{6} p^{2} \\
\Delta p=b_{1}+b_{2} l+b_{3} p+b_{4} l p+b_{5} l^{2}+b_{6} p^{2}
\end{array}\right.
$$

where $(\Delta l, \Delta p)$ are the offset vectors of the homologous points, $a_{i}, b_{i}$ are the polynomial coefficients, $(l, p)$ are the pixel coordinates of master image.

Using the least squares method, the coefficients of two-dimensional polynomial can be estimated and the co-registration model between the master and slave images is obtained. The derived co-registration model can be further used to resample and align the slave image to the master image with improved accuracy.

\section{EXPERIMENTAL RESULTS}

To validate the reliability and robustness of the proposed algorithm for SAR image co-registration, the ALOS 
PALSAR images covering Longmenshan mountainous area in southwestern China are used in the study.

Two ALOS PALSAR images acquired respectively on $09 / 08 / 2009$ and on $10 / 24 / 2009$ are used to form the interferometric pair. The center latitude and longitude of the acquisition scenes are $104.01^{\circ} \mathrm{E}$ and $31.46^{\circ} \mathrm{N}$. The giant Ms8.0 Wenchuan earthquake occurred in the area on May 122008 .

The coarse co-registration processing was first carried out based on the satellite orbital state vectors and the set of geometric equations. The central pixel coordinate in the master image is $(12400,2500)$. With the coarse coordinate offset vectors, the matched point coordinate in the slave image is determined with the pixel position (12363.3, 2476.2). The pixel coordinate offset is almost $(-37,-24)$ in the azimuth and range directions.

More feature points evenly distributed in the master image are selected to calculate the cross-correlation. The coordinate offset vector between the master and slave image was adjusted to a more reliable value through discrete maximum coherence search. The co-registration accuracy from the discrete windows computation reaches about one pixel.

In search of the matching points with sub-pixel accuracy, the previous moving surface fitting method is employed to obtain the continuous coherence field. From the fast solution for the set of differential equations, the accurate positions of homologous points were found.

Fig. 1 shows the offset vector field of matched points, which are calculated based on the moving coherence surface fitting method. The offset vectors represent the good consistency in the magnitude and direction.

Fig. 2 shows the coherence map in the study area. Two significant coherence difference areas are found along the northeast-southwest direction. The dark and light gray in the map indicate respectively the low and high coherence. The mountainous area in the northwest presents the low coherence, and the plain area in the southeast presents the high coherence.

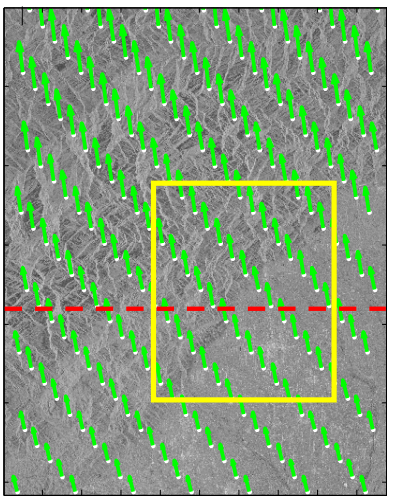

Fig .1 Offset vector field

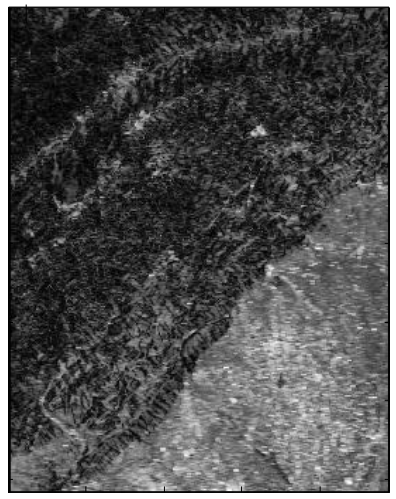

Fig. 2 Coherence map
Fig. 3 shows the coherence difference from two different co-registration methods, the moving coherence surface fitting and the coherence interpolation method. It indicates that the coherence from the surface fitting method is slightly higher than that from the interpolation method. The statistics of coherence difference shows that the coherence improvement was achieved with almost 5\% at high correlation pixels.

A clear interferogram shown as Fig. 4 (corresponding to the yellow rectangle area in Fig. 1) was derived despite of the steep terrain in the region and the long time interval (46 days) of two SAR acquisitions. On the one hand, it is due to the less temporal decorrelation impact on the long wavelength L-band PALSAR data. On the other hand, the image co-registration and resampling approaches with high accuracy are employed to mitigate the coherence loss, resulting in the high quality interferogram.

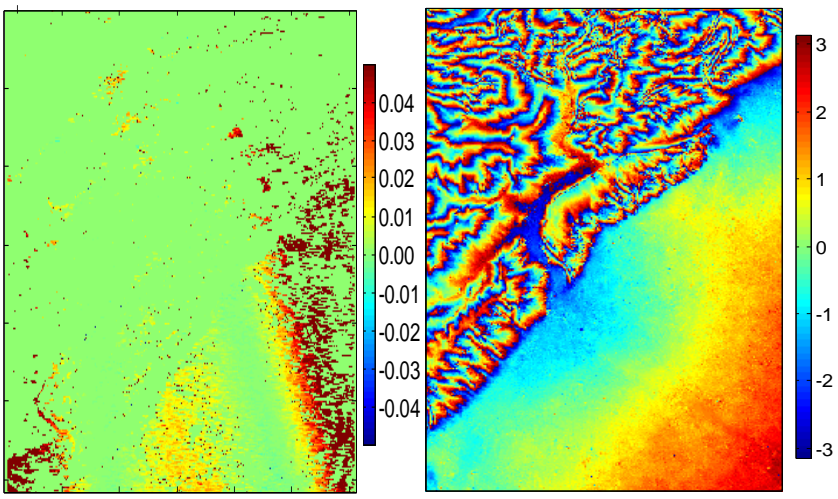

Fig.3 Coherence difference Fig. 4 Interferogram map

In order to further assess the accuracy of SAR image co-registration, we compare the coordinates offset of matched positions in the azimuth and range direction at the homologous points. The pixels marked along the red line in Fig. 1 were selected to make the coordinate comparison between the coherence surface fitting method and the coherence interpolation method. The derived coordinates offset in the azimuth and range direction are respectively shown in Fig. 5 and Fig. 6. The horizontal axis indicates the pixel series along the red line (4500 pixels), and the vertical axis indicates the coordinate offset of the matched points.

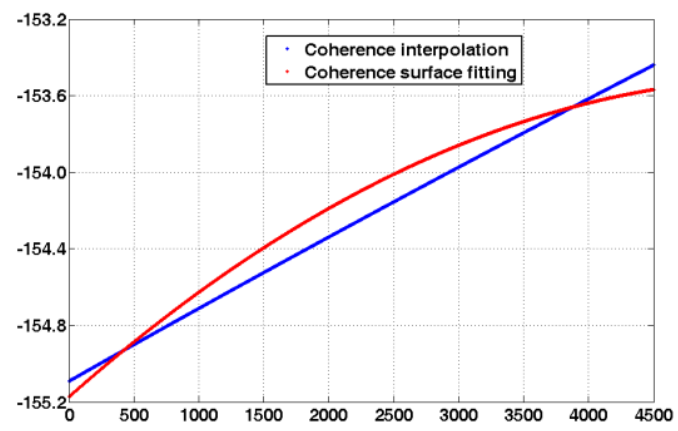

Fig. 5 Coordinate offset in azimuth direction 


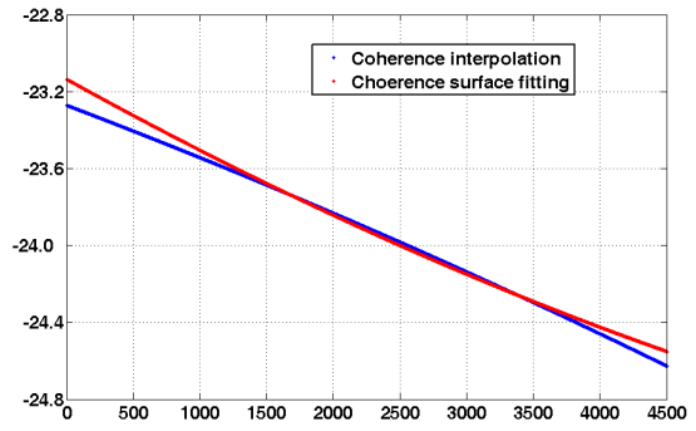

Fig. 6 Coordinate offset in range direction

The statistics at the homologous points shows that the coordinate offset differences are in the range $(-0.07 \sim 0.15)$ and $(-0.02 \sim 0.03)$ pixels in the azimuth and range direction respectively. The root mean square error (RMSE) of the co-registration coordinate difference is respectively 0.076 pixels and 0.011 pixels in the azimuth and range direction. The comparison results validate the reliability and robustness of the proposed algorithm for SAR image co-registration.

\section{CONCLUSIONS}

In order to mitigate the coherence loss due to discrete windows search in SAR image co-registration, the method of moving coherence surface fitting is proposed to detect accurate positions of homologous points. The experimental results show that the co-registration accuracy of SAR images reaches $(0.1 \sim 0.01)$ pixels and the coherence in the testing area are improved with about 5\%. The quality improvement of interferogram validates the reliability and robustness of the proposed method for SAR image co-registration.

\section{ACKNOWLEDGEMENTS}

This work was jointly supported by the Natural Science Foundation of China (Grant Nos. 41072220, 51178404) and Fundamental Research Funds for the Central Universities (Grant Nos. SWJTU09CX010, SWJTU11ZT13).

\section{REFERENCES}

[1] B. M. Kampes, R. F. Hanssen. Perski Z. Radar Interferometry with Public Domain Tools. Proc. FRINGE 2003 Workshop, December 1-5, 2003, Frascati, Italy.

[2] H. A. Zebker , R. M. Goldstein. Topographic Mapping from Interferometric Synthetic Aperture Radar Observation. Journal of Geophysical research, vol. 91, pp. 4993-4999, 1986.

[3] D. O. Nitti, R. F. Hanssen, A. Refice, F. Bovenga, G. Milillo and R. Nutricato. Evaluation of DEM-assisted SAR Co-registration. In proceedings of SPIE Remote Sensing, Cardiff, Wales, United Kingdom, 2008.

[4] LI Zhili, LIU Guoxiang, DING Xiaoli. Exploring the Generation of Digital Elevation Models From Same-side ERS SAR Images: Topographic and Temporal Effects. Photogrammetric Record, vol. 10, no. 113, pp. 1-17, 2006.

[5] A. Ferretti, C. Prati and F. Rocca. Permanent Scatters in SAR Interferometry. IEEE Transactions on Geoscience and Remote Sensing, vol. 39, no. 1, pp. 8-20, 2001.

[6] R. Scharroo, P. Visser. Precise Orbit Determination and Gravity Field Improvement for the ERS Satellites. Journal of Geophysical Research, vol. 103(C4), pp. 8113-8127, 1998.

[7] ZENG Qiming, Xie Xuetong. A FFT-based Complex Correlation Function Method Applied to Interferometric Complex Image Coregistration (in Chinese). Acta Geodaetica et Carto graphica Sinica, 2004, 33(2):128-131.

[8] LIU Guoxiang, DING Xiaoli, LI Zhilin. Coregistration of satellite SAR complex images (in Chinese). Acta Geodaetica et Carto Graphica Sinica, 2001, 30(1):60-66. 